\title{
WHEN IS THE HERMITIAN/SKEW-HERMITIAN PART OF A MATRIX A POTENT MATRIX?*
}

\author{
DIJANA ILIŠEVIĆ† AND NÉSTOR THOME
}

\begin{abstract}
This paper deals with the Hermitian $H(A)$ and skew-Hermitian part $S(A)$ of a complex matrix $A$. We characterize all complex matrices $A$ such that $H(A)$, respectively $S(A)$, is a potent matrix. Two approaches are used: characterizations of idempotent and tripotent Hermitian matrices of the form $\left[\begin{array}{cc}X & Y^{*} \\ Y & 0\end{array}\right]$, and a singular value decomposition of $A$. In addition, a relation between the potency of $H(A)$, respectively $S(A)$, and the normality of $A$ is also studied.
\end{abstract}

Key words. Hermitian part, skew-Hermitian part, potent matrix, normal matrix.

AMS subject classifications. 15B57, 15A23.

1. Introduction. Throughout, $A^{*}$ denotes the conjugate transpose of the complex matrix $A$. Motivated by an application to statistics related to the chi-square distribution, Khatri [19] studied the case when the Hermitian part $H(A)=\frac{1}{2}\left(A+A^{*}\right)$ of a complex matrix $A \in \mathbb{C}^{n \times n}$, with the same rank as $A$, is idempotent. Groß [14] extended this result by relaxing the assumption on the rank. The purpose of our paper is to generalize these results concerning $H(A)$ as well as to study the corresponding problem for the skew-Hermitian part $S(A)=\frac{1}{2}\left(A-A^{*}\right)$ of $A$.

General considerations on Hermitian and skew-Hermitian elements in a division ring with an involution were given in [13], where the relation between certain powers was analyzed.

In [22], the Hermitian and skew-Hermitian parts of a matrix are used to give some inequalities involving norms, Hadamard products and submatrices. These results have been applied to derive a bound on the backward and forward error of the solution of

\footnotetext{
* Received by the editors on November 11, 2011. Accepted for publication on March 24, 2012. Handling Editor: Oskar Maria Baksalary.

$\dagger$ Department of Mathematics, University of Zagreb, Bijenička 30, 10000 Zagreb, Croatia (ilisevic@math.hr). Supported by the Ministry of Science, Educations and Sports of the Republic of Croatia (Grant 037-0372784-2757).

${ }^{\ddagger}$ Instituto Universitario de Matemática Multidisciplinar, Universitat Politècnica de València, Camino de Vera s/n, 46022, València, Spain (njthome@mat.upv.es). Supported by the Ministry of Education of Spain (Grant DGI MTM2010-18228), the Universidad Nacional de La Pampa, Argentina (Grant Resol. N. 049/11), and the Ministry of Education of Argentina (PPUA, Grant Resol. 228, SPU, 14-15-222).
} 
linear systems $A x=b$ (with $H(A)$ positive definite).

Furthermore, from a numerical point of view, the Hermitian and skew-Hermitian parts of a matrix were used for solving saddle-point problems, image restoration, etc. $[3,6,11,17,20,21]$. In [6], Bai, Golub and $\mathrm{Ng}$ introduced the Hermitian and skew-Hermitian splitting (HSS) iteration method and discussed its unconditional convergence property; see also [3] and [4]. The HSS iteration method was further generalized to the normal and skew-Hermitian splitting (NSS) iteration method in [7] and to the positive definite and skew-Hermitian splitting (PSS) iteration method in [5]. Benzi and Golub [12] developed the HSS iteration method to the saddle-point problems; see also [11] and [8].

In this paper we first characterize all complex matrices $A \in \mathbb{C}^{n \times n}$ satisfying one of the following conditions:

- $H(A)^{k+1}=H(A)$ for some $k \in \mathbb{N}$,

- $S(A)^{k+1}=S(A)$ for some $k \in \mathbb{N}$.

Recall that a square matrix $X$ is said to be a potent matrix (more precisely, a $\{k+1\}$ potent matrix) if there exists $k \in \mathbb{N}$ such that $X^{k+1}=X$. If a $\{k+1\}$-potent matrix $X$ is nonsingular then $X^{k}=I$; such matrices are said to be of finite order. Some properties of $\{k+1\}$-potent matrices can be found in [15, 23, 24]. Thus, in other words, we characterize all complex square matrices whose Hermitian/skew-Hermitian parts are potent matrices (in particular, matrices of finite order). In addition, a singular value decomposition (SVD) is used to present another characterization; one example illustrates this result. Later, we shall state a relation between the potency of $H(A)$ or $S(A)$ and the normality of $A$.

The next result, which can be easily checked, will be useful in further considerations.

Lemma 1.1. Let $X \in \mathbb{C}^{n \times n}$ be a $\{k+1\}$-potent matrix where $k \in \mathbb{N}$.

(a) If $X$ is a Hermitian matrix then $X^{3}=X$ when $k$ is even, and $X^{2}=X$ when $k$ is odd.

(b) If $X$ is a skew-Hermitian matrix then $X^{3}=-X$ when $k$ is a multiple of 4, and $X=0$ when $k$ is not a multiple of 4 .

Part (a) of Lemma 1.1 assures that the analysis of the idempotency and tripotency of the Hermitian part of a complex matrix solves the proposed problem for every arbitrary power of the Hermitian part. Analogously, part (b) of Lemma 1.1 reduces the study of the $\{k+1\}$-potency of the skew-Hermitian part to the case when $k$ is a multiple of 4 .

Notice that in the case when a $\{k+1\}$-potent matrix $X \in \mathbb{C}^{n \times n}$ is nonsingular, 
the analysis of such matrices is reduced to the analysis of involutory matrices (for Hermitian matrices) and skew-involutory matrices (for skew-Hermitian matrices).

Obviously, $H(\mathrm{i} A)=\mathrm{i} S(A)$ for all square matrices $A$, where i denotes the imaginary unit. Therefore, the study of the $\{k+1\}$-potency of $S(A)$ reduces to the study of the $\{k+1\}$-potency of $-\mathrm{i} H(\mathrm{i} A)$.

2. Powers of the Hermitian and skew-Hermitian parts. Let $\mathcal{N}(A)$ be the null space of a matrix $A \in \mathbb{C}^{n \times n}, \mathcal{N}(A)^{\perp}$ its orthogonal complement and $\mathcal{R}(A)$ the range of $A$. We start this section with some interesting particular cases.

(1) Hermitian matrices: $A^{*}=A$. In this case,

$$
H(A)^{k+1}=H(A) \quad \Leftrightarrow \quad A^{k+1}=A \quad \Leftrightarrow \quad A^{2}=A \text { or } A^{3}=A
$$

(2) Normal matrices: $A A^{*}=A^{*} A$. In this case, $A=U D U^{*}$ for some unitary matrix $U$ and some diagonal matrix $D$. Then $H(A)=U(\operatorname{Re}(D)) U^{*}$, where $\operatorname{Re}(D)=\frac{1}{2}\left(D+D^{*}\right)$, that is, $\operatorname{Re}(D)$ is a diagonal matrix with diagonal entries consisting of the real parts of elements in $\sigma(A)$. So,

$$
H(A)^{k+1}=H(A) \Leftrightarrow \operatorname{Re}(D)^{k+1}=\operatorname{Re}(D) \Leftrightarrow \operatorname{Re}\left(d_{i}\right) \in\{-1,0,1\}, \forall d_{i} \in \sigma(A) .
$$

(3) EP matrices: $\mathcal{R}(A)=\mathcal{R}\left(A^{*}\right)$, which is equivalent to $A=U\left[\begin{array}{ll}C & O \\ O & O\end{array}\right] U^{*}$ where $U$ is unitary and $C$ is a nonsingular matrix. In this case,

$$
H(A)=U\left[\begin{array}{cc}
H(C) & O \\
O & O
\end{array}\right] U^{*}
$$

Since $H(C)$ is Hermitian, $H(C)=U_{C} D_{C} U_{C}^{*}$, where $U_{C}$ is unitary and $D_{C}$ is diagonal with real entries. So,

$$
H(A)^{k+1}=H(A) \Leftrightarrow D_{C}^{k+1}=D_{C} \Leftrightarrow H(C)^{2}=H(C) \text { or } H(C)^{3}=H(C) .
$$

(4) Nonsingular matrices: If $A$ is nonsingular then $A^{*}$ is also nonsingular. Hence, $\mathcal{R}(A)=\mathbb{C}^{n}=\mathcal{R}\left(A^{*}\right)$, that is, $A$ is an $E P$ matrix. Notice that the matrix $C$ in (3) has the same size as $A$. Thus,

$$
H(A)^{k+1}=H(A) \Leftrightarrow H(A)^{2}=H(A) \text { or } H(A)^{3}=H(A)
$$

It is well known that these sets of matrices satisfy:

$$
\text { Hermitian matrices } \subset \text { Normal matrices } \subset E P \text { matrices. }
$$


We want to extend these observations to a general matrix $A$. The next lemma will be crucial in our characterizations of the $\{k+1\}$-potency of the Hermitian and skewHermitian parts (cf. [16, Corollary 6]).

Lemma 2.1. Let $A \in \mathbb{C}^{n \times n}$ be a matrix of rank $r<n$. Then there exist $T \in \mathbb{C}^{r \times r}$, $Y \in \mathbb{C}^{(n-r) \times r}$, and a unitary matrix $U \in \mathbb{C}^{n \times n}$ such that

$$
A=U\left[\begin{array}{ll}
T & 0 \\
Y & 0
\end{array}\right] U^{*}
$$

Proof. Let $\left\{v_{1}, \ldots, v_{r}\right\}$ be an orthonormal basis of $\mathcal{N}(A)^{\perp}$ and let $\left\{v_{r+1}, \ldots, v_{n}\right\}$ be an orthonormal basis of $\mathcal{N}(A)$. Let $U$ be the unitary matrix whose columns are the vectors $v_{1}, \ldots, v_{r}, v_{r+1}, \ldots, v_{n}$. The block of the first $r$ columns will be denoted by $U_{1}$ and the block of the last $n-r$ columns will be denoted by $U_{2}$, that is, $U_{1}=$ $\left[\begin{array}{lll}v_{1} & \cdots & v_{r}\end{array}\right]$ and $U_{2}=\left[\begin{array}{lll}v_{r+1} & \cdots & v_{n}\end{array}\right]$. Then we get

$$
U^{*} A U=U^{*} A\left[\begin{array}{ll}
U_{1} & U_{2}
\end{array}\right]=\left[\begin{array}{ll}
U^{*} A U_{1} & U^{*} A U_{2}
\end{array}\right]=\left[\begin{array}{ll}
U^{*} A U_{1} & 0
\end{array}\right] .
$$

Now, we can write

$$
A=U\left[\begin{array}{ll}
T & 0 \\
Y & 0
\end{array}\right] U^{*}
$$

where $T \in \mathbb{C}^{r \times r}$ and $Y \in \mathbb{C}^{(n-r) \times r}$.

The expression (2.1) for $A$ is also valid when $A$ is nonsingular (in this case $T$ is an $n \times n$ nonsingular matrix and $Y$ and the null blocks are absent). Notice that $\mathcal{N}(A)^{\perp}=\mathcal{R}\left(A^{*}\right)$. Let us also remark that $T$ and $Y$ in (2.1) can be chosen so that they satisfy a useful relation [16, Corollary 6], providing a powerful tool for characterization of various classes of matrices [9]. However, we choose $T$ and $Y$ under the condition that $Y^{*} Y$ is diagonal, as we state in the following remark (in this case, the nice relation from [16, Corollary 6] is not preserved).

REMARK 2.2. Without loss of generality, we can assume that the matrix $Y \in$ $\mathbb{C}^{(n-r) \times r}$ from (2.1) is such that $Y^{*} Y$ is diagonal with positive entries in the first $m \leq r$ diagonal positions, where $m=\operatorname{rank}(Y)$; if $m=0$ then $Y^{*} Y=0$. Namely, there exist a unitary matrix $V \in \mathbb{C}^{r \times r}$ and a diagonal matrix $D \in \mathbb{C}^{r \times r}$, with positive entries in the first $m$ diagonal positions, such that $Y^{*} Y=V D V^{*}$. Let $S=V^{*} T V \in \mathbb{C}^{r \times r}$, $Z=Y V \in \mathbb{C}^{(n-r) \times r}$ and $W=U \operatorname{diag}(V, I) \in \mathbb{C}^{n \times n}$. Then $Z^{*} Z=V^{*} Y^{*} Y V$, the matrix $W$ is unitary, and

$A=U\left[\begin{array}{ll}T & 0 \\ Y & 0\end{array}\right] U^{*}=U\left[\begin{array}{ll}V & 0 \\ 0 & I\end{array}\right]\left[\begin{array}{cc}V^{*} T V & 0 \\ Y V & 0\end{array}\right]\left[\begin{array}{cc}V^{*} & 0 \\ 0 & I\end{array}\right] U^{*}=W\left[\begin{array}{cc}S & 0 \\ Z & 0\end{array}\right] W^{*}$. 
Thus, it is sufficient to replace $U$ with $W, T$ with $V^{*} T V$, and $Y$ with $Y V$ in (2.1).

REMARK 2.3. If $Y=0$ (or $Y$ and the null blocks are absent), then $T$ is a nonsingular matrix, that is, $A$ is an EP matrix. In this case, without loss of generality, we can assume that the matrix $T$ is an upper triangular matrix. In fact, the Schur's triangularization theorem (cf. [18]), applied to $T$, assures that there exists a unitary matrix $V_{1}$ such that $T=V_{1} T_{1} V_{1}^{*}$, where $T_{1}$ is an upper triangular nonsingular matrix, so we have

$$
A=V_{2}\left[\begin{array}{cc}
T_{1} & 0 \\
0 & 0
\end{array}\right] V_{2}^{*}, \quad \text { where } V_{2}=U\left[\begin{array}{cc}
V_{1} & 0 \\
0 & I
\end{array}\right] \text { is unitary }
$$

First let us consider the question of when a special matrix is idempotent, tripotent, or involutory. This special matrix $X$ is a Hermitian matrix of the saddle-point type (such matrices are used to solve the saddle-point problem [4]). It is assumed to be Hermitian because it will be used in the proof of Theorem 2.6 setting

$$
X=U^{*} H(A) U=\left[\begin{array}{cc}
H(T) & \frac{1}{2} Y^{*} \\
\frac{1}{2} Y & 0
\end{array}\right],
$$

with $U, T, Y$ as in Lemma 2.1 and Remark 2.2.

Lemma 2.4. Let $X \in \mathbb{C}^{n \times n}$ be a Hermitian matrix and suppose that there exist $r<n$, a Hermitian matrix $A \in \mathbb{C}^{r \times r}$, and a matrix $B \in \mathbb{C}^{(n-r) \times r}$ of rank $m$ such that $B^{*} B$ is diagonal with positive entries in the first $m$ diagonal positions and

$$
X=\left[\begin{array}{cc}
A & B^{*} \\
B & 0
\end{array}\right]
$$

Then the following holds.

(a) $X^{2}=X$ if and only if $A^{2}=A$ and $B=0$.

(b) $X^{3}=X$ if and only if one of the following holds:

(i) $n=2 r, A=0$, and $B$ is unitary,

(ii) $A^{3}=A$ and $B=0$,

(iii) $0<m<r$ and there are a matrix $W \in \mathbb{C}^{(r-m) \times m}$, a Hermitian matrix $Z \in \mathbb{C}^{(r-m) \times(r-m)}$, and a matrix $B_{1} \in \mathbb{C}^{(n-r) \times m}$ such that

$$
A=\left[\begin{array}{cc}
0 & W^{*} \\
W & Z
\end{array}\right], \quad B=\left[\begin{array}{ll}
B_{1} & 0
\end{array}\right]
$$

with $Z^{3}=Z, Z W=0$, and $B_{1}^{*} B_{1}=I-W^{*} W$ which is a positive definite diagonal matrix.

(c) $X^{2}=I$ if and only if one of the following holds: 
(i) $n=2 r, A=0$, and $B$ is unitary,

(ii) $0<m=n-r<r$, and there are a Hermitian matrix $Z \in \mathbb{C}^{(r-m) \times(r-m)}$ and a unitary matrix $B_{1} \in \mathbb{C}^{m \times m}$ such that $Z^{2}=I$,

$$
A=\left[\begin{array}{ll}
0 & 0 \\
0 & Z
\end{array}\right], \quad \text { and } \quad B=\left[\begin{array}{ll}
B_{1} & 0
\end{array}\right] .
$$

Proof. (a) We have

$$
X^{2}=\left[\begin{array}{cc}
A^{2}+B^{*} B & A B^{*} \\
B A & B B^{*}
\end{array}\right]
$$

Thus $X^{2}=X$ if and only if $A^{2}+B^{*} B=A, B A=B$, and $B B^{*}=0$. Since $B B^{*}=0$ if and only if $B=0$, we get $A^{2}=A$.

(b) We have

$$
X^{3}=\left[\begin{array}{cc}
A^{3}+B^{*} B A+A B^{*} B & A^{2} B^{*}+B^{*} B B^{*} \\
B A^{2}+B B^{*} B & B A B^{*}
\end{array}\right] .
$$

Then $X^{3}=X$ if and only if the following conditions hold:

(C1) $A^{3}+B^{*} B A+A B^{*} B=A$,

(C2) $A^{2} B^{*}+B^{*} B B^{*}=B^{*}$,

(C3) $B A B^{*}=0$.

Let us remark that (C3) can be obtained from (C1) and (C2): subtracting the equalities obtained multiplying $(\mathrm{C} 1)$ by $B^{*}$ from the right and multiplying (C2) by $A$ from the left, we first conclude $B^{*} B A B^{*}=0$, then $\left(B A B^{*}\right)^{*}\left(B A B^{*}\right)=0$, and finally $B A B^{*}=0$.

If $B$ is nonsingular, we get (i); if $B$ is zero, we get (ii). Let us assume $0<m<r$ and let $D=B^{*} B$. By the assumption on $B$, we get that $D=\operatorname{diag}(E, 0)$, with $E \in$ $\mathbb{C}^{m \times m}$ a positive definite diagonal matrix. Let us observe the following conditions:

(C1') $A^{3}+D A+A D=A$,

(C2') $A^{2} D+D^{2}=D$

(C3') $D A D=0$.

Clearly, (C1) and (C1') are the same conditions. Notice that (C2) $\Leftrightarrow\left(\mathrm{C} 2{ }^{\prime}\right)$ and (C3) $\Leftrightarrow\left(\mathrm{C} 3{ }^{\prime}\right)$. Namely, (C2) $\Rightarrow\left(\mathrm{C} 2{ }^{\prime}\right)$ and $(\mathrm{C} 3) \Rightarrow\left(\mathrm{C} 3{ }^{\prime}\right)$ are obvious. Conversely, if (C2') holds and we set $M=A^{2} B^{*}+B^{*} B B^{*}-B^{*}$, then (C2') yields $M M^{*}=0$, hence $M=0$; if (C3') holds and we set $N=B A B^{*}$, then (C3') yields $N^{*} N N^{*}=0$, which implies $N=0$. Therefore, $X^{3}=X$ if and only if the conditions (C1'), (C2') and (C3') hold. 
Let

$$
A=\left[\begin{array}{cc}
P & W^{*} \\
W & Z
\end{array}\right]
$$

with $P \in \mathbb{C}^{m \times m}$ Hermitian, $W \in \mathbb{C}^{(r-m) \times m}$, and $Z \in \mathbb{C}^{(r-m) \times(r-m)}$ Hermitian. Since $D=\operatorname{diag}(E, 0)$, with $E \in \mathbb{C}^{m \times m}$, we conclude that (C3') is equivalent to $E P E=0$. Since $E$ is invertible, $P=0$. The condition (C2') is equivalent to $W^{*} W E+E^{2}=E$ and $Z W E=0$. Since $E$ is invertible, we conclude $W^{*} W+E=I$ and $Z W=0$. Furthermore, (C1') is equivalent to

$$
\left[\begin{array}{cc}
W^{*} Z W & W^{*} W W^{*}+W^{*} Z^{2}+E W^{*} \\
W W^{*} W+Z^{2} W+W E & W W^{*} Z+Z W W^{*}+Z^{3}
\end{array}\right]=\left[\begin{array}{cc}
0 & W^{*} \\
W & Z
\end{array}\right] .
$$

Taking into account $E=I-W^{*} W, Z W=0$, and $W^{*} Z=0$, we conclude $Z^{3}=Z$. Let us partition

$$
B=\left[\begin{array}{ll}
B_{1} & B_{2}
\end{array}\right]
$$

where $B_{1} \in \mathbb{C}^{(n-r) \times m}$ and $B_{2} \in \mathbb{C}^{(n-r) \times(r-m)}$. Comparing the product $B^{*} B$ computed via (2.3) with $B^{*} B=\operatorname{diag}\left(I-W^{*} W, 0\right)$, it is easy to conclude that $B_{2}=0$ and $B_{1}^{*} B_{1}=I-W^{*} W$. Hence, (iii) holds.

It can be easily verified that any of (i), (ii), (iii) implies $X^{3}=X$.

(c) First notice that $X^{2}=I$ if and only if $A^{2}+B^{*} B=I, B A=0$, and $B B^{*}=I$. If $B$ is nonsingular, then $A=0$ and $B^{*} B=I$. The converse is obvious.

Assume $0<m<r$. Since $X^{2}=I$ implies $X^{3}=X$, we may apply (iii) from (b). Hence,

$$
A=\left[\begin{array}{cc}
0 & W^{*} \\
W & Z
\end{array}\right], \quad B=\left[\begin{array}{ll}
B_{1} & 0
\end{array}\right]
$$

with $Z$ Hermitian, $Z^{3}=Z, Z W=0$, and $B_{1}^{*} B_{1}=I-W^{*} W$ which is a positive definite diagonal matrix. However, we have

$$
\begin{gathered}
I=A^{2}+B^{*} B=\left[\begin{array}{cc}
I & 0 \\
0 & W W^{*}+Z^{2}
\end{array}\right], \\
0=B A=\left[\begin{array}{ll}
0 & B_{1} W^{*}
\end{array}\right], \quad I=B B^{*}=B_{1} B_{1}^{*} .
\end{gathered}
$$

This implies $W W^{*}+Z^{2}=I$ and $B_{1} W^{*}=0$. Then $B_{1}^{*} B_{1} W^{*}=0$ as well. Since $B_{1}^{*} B_{1}$ is a positive definite diagonal matrix, it is nonsingular, so $W=0$. This yields $Z^{2}=I$ 
and $B_{1}^{*} B_{1}=I$. Since $\operatorname{rank}\left(B_{1}^{*} B_{1}\right)=\operatorname{rank}\left(B_{1} B_{1}^{*}\right)$, we conclude $m=n-r$, hence $B_{1}$ is unitary. Thus, (ii) holds. It is easy to check that (ii) implies $X^{2}=I$.

REMARK 2.5. When (iii) in item (b) of Lemma 2.4 holds, then $B_{1}$ has nonzero orthogonal columns (because $B_{1}^{*} B_{1}$ is diagonal positive definite) and the matrix $\left[\begin{array}{l}B_{1} \\ W\end{array}\right]$ is a partial isometry (that is, it coincides with its Moore-Penrose inverse) because

$$
\left[\begin{array}{l}
B_{1} \\
W
\end{array}\right]^{*}\left[\begin{array}{l}
B_{1} \\
W
\end{array}\right]=I .
$$

It is easy to see that the converse is also true.

The desired results on the $\{k+1\}$-potency of the Hermitian/skew-Hermitian parts of a complex matrix now immediately follow from Lemma 2.1, Lemma 1.1, and Lemma 2.4 .

Theorem 2.6. Let $A \in \mathbb{C}^{n \times n}$ and $k \in \mathbb{N}$. Then:

(I) $H(A)^{k+1}=H(A)$ if and only if there exists a unitary matrix $U \in \mathbb{C}^{n \times n}$ such that $A$ has the form (2.1) with $Y \in \mathbb{C}^{(n-r) \times r}$ of rank $m$ such that $Y^{*} Y$ is diagonal with positive entries in the first $m$ diagonal positions, and

(a) if $k$ is odd then $Y=0$ and $H(T)^{2}=H(T)$,

(b) if $k$ is even then one of the following holds:

(i) $n=2 r, H(T)=0$, and $\frac{1}{2} Y$ is unitary,

(ii) $Y=0$ and $H(T)^{3}=H(T)$,

(iii) $0<m<r$ and there exist $W \in \mathbb{C}^{(r-m) \times m}$, a Hermitian matrix $Z \in \mathbb{C}^{(r-m) \times(r-m)}$, and a matrix $Y_{1} \in \mathbb{C}^{(n-r) \times m}$ such that

$$
H(T)=\left[\begin{array}{cc}
0 & W^{*} \\
W & Z
\end{array}\right], \quad Y=\left[\begin{array}{ll}
Y_{1} & 0
\end{array}\right],
$$

with $Z^{3}=Z, Z W=0$, and $Y_{1}^{*} Y_{1}=4\left(I-W^{*} W\right)$ which is a positive definite diagonal matrix.

(II) $S(A)^{k+1}=S(A)$ if and only if there exists a unitary matrix $U \in \mathbb{C}^{n \times n}$ such that $A$ has the form (2.1) with $Y \in \mathbb{C}^{(n-r) \times r}$ of rank $m$ such that $Y^{*} Y$ is diagonal with positive entries in the first $m$ diagonal positions, and

(a) if $k$ is not a multiple of 4 then $S(A)=0$,

(b) if $k$ is a multiple of 4 then one of the following holds:

(i) $n=2 r, S(T)=0$, and $\frac{1}{2} Y$ is unitary,

(ii) $Y=0$ and $S(T)^{3}=-S(T)$,

(iii) $0<m<r$ and there exist $W \in \mathbb{C}^{(r-m) \times m}$, a skew-Hermitian matrix $Z \in \mathbb{C}^{(r-m) \times(r-m)}$, and a matrix $Y_{1} \in \mathbb{C}^{(n-r) \times m}$ such that

$$
S(T)=\left[\begin{array}{cc}
0 & -W^{*} \\
W & Z
\end{array}\right], \quad Y=\left[\begin{array}{ll}
Y_{1} & 0
\end{array}\right],
$$


with $Z^{3}=-Z, Z W=0$, and $Y_{1}^{*} Y_{1}=4\left(I-W^{*} W\right)$ which is a positive definite diagonal matrix.

Proof. Application of Lemma 1.1 and Lemma 2.4 to the matrix

$$
U^{*} H(A) U=\left[\begin{array}{cc}
H(T) & \frac{1}{2} Y^{*} \\
\frac{1}{2} Y & 0
\end{array}\right]
$$

proves part (I) of the theorem. Part (II) follows from $S(A)=-\mathrm{i} H(\mathrm{i} A)$.

EXAMPLE 2.7. Let

$$
A=\left[\begin{array}{ll}
\mathrm{i} & 0 \\
2 & 0
\end{array}\right] .
$$

Then, using the notation of Lemma 2.1, $n=2, r=\operatorname{rank}(A)=1, T=[\mathrm{i}]$, and $Y=[2]$. This implies $n=2 r, H(T)=0$, and $\frac{1}{2} Y=[1]$ is unitary, which corresponds to case (i) in (I)-(b) of Theorem 2.6. Then $H(A)^{k+1}=H(A)=\left[\begin{array}{ll}0 & 1 \\ 1 & 0\end{array}\right]$ for all even $k$. Since $Y \neq 0$, we conclude $H(A)^{k+1} \neq H(A)$ for all odd $k$.

The previous example shows that there exists a matrix $A$ such that $H(A)^{k+1}=$ $H(A)$ for all even $k$, but $H(A)^{k+1} \neq H(A)$ for all odd $k$. However, if $H(A)^{k+1}=$ $H(A)$ for some odd $k \in \mathbb{N}$ then $H(A)^{k+1}=H(A)$ for all $k \in \mathbb{N}$. Namely, if there exists an odd $k$ such that $H(A)^{k+1}=H(A)$ then $H(A)^{2}=H(A)$ by Lemma 1.1, thus $H(A)^{k+1}=H(A)$ for all odd $k$. This implies $H(A)^{3}=H(A)^{2}=H(A)$, hence $H(A)^{k+1}=H(A)$ for all even $k$ as well.

EXAMPLE 2.8. Let

$$
T=\left[\begin{array}{rr}
1 & 1 \\
-1 & 0
\end{array}\right], \quad Y=0_{m \times 2}, \quad \text { and } \quad A=\left[\begin{array}{cc}
T & 0_{2 \times m} \\
Y & 0_{m \times m}
\end{array}\right],
$$

where $0_{r \times c}$ denotes the zero matrix of size $r \times c$, and $m \in \mathbb{N}$.

It is easy to see that

$$
H(T)=\left[\begin{array}{ll}
1 & 0 \\
0 & 0
\end{array}\right]
$$

hence $H(T)^{2}=H(T)$. Then $H(A)^{k+1}=H(A)$ for all $k \in \mathbb{N}$.

When $X$ is nonsingular, the problem of the $\{k+1\}$-potency of $X$ is reduced to $X^{k}=I$. If $X$ is the Hermitian/skew-Hermitian part of a matrix $A$ then part (c) of Lemma 2.4 is applicable and the following results can be easily obtained.

Notice that in this case the condition $Y=0$ can be only formally fulfilled, that is, it holds if and only if $Y$ and the null blocks are absent.

Theorem 2.9. Let $A \in \mathbb{C}^{n \times n}$ and $k \in \mathbb{N}$. Then: 
(I) $H(A)^{k}=I$ if and only if there exists a unitary matrix $U \in \mathbb{C}^{n \times n}$ such that $A$ has the form (2.1) with $Y \in \mathbb{C}^{(n-r) \times r}$ of rank $m$ such that $Y^{*} Y$ is diagonal with positive entries in the first $m$ diagonal positions, and

(a) if $k$ is odd then $Y$ and the null blocks are absent and $H(T)=I$,

(b) if $k$ is even then one of the following holds:

(i) $Y$ and the null blocks are absent and $H(T)^{2}=I$,

(ii) $n=2 r, H(T)=0$, and $\frac{1}{2} Y$ is unitary,

(iii) $0<m=n-r<r$, and there exist a Hermitian matrix $Z \in$ $\mathbb{C}^{(r-m) \times(r-m)}$, and a matrix $Y_{1} \in \mathbb{C}^{m \times m}$ such that $Z^{2}=I$,

$$
H(T)=\left[\begin{array}{ll}
0 & 0 \\
0 & Z
\end{array}\right], \quad Y=\left[\begin{array}{ll}
Y_{1} & 0
\end{array}\right],
$$

and $\frac{1}{2} Y_{1}$ is unitary.

(II) $S(A)^{k}=I$ if and only if $k$ is a multiple of 4 and there exists a unitary matrix $U \in \mathbb{C}^{n \times n}$ such that $A$ has the form (2.1) with $Y \in \mathbb{C}^{(n-r) \times r}$ of rank $m$ such that $Y^{*} Y$ is diagonal with positive entries in the first $m$ diagonal positions, and one of the following holds:

(i) $Y$ and the null blocks are absent and $S(T)^{2}=-I$,

(ii) $n=2 r, S(T)=0$, and $\frac{1}{2} Y$ is unitary,

(iii) $0<m=n-r<r$ and there exist a skew-Hermitian matrix $Z \in$ $\mathbb{C}^{(r-m) \times(r-m)}$, and a matrix $Y_{1} \in \mathbb{C}^{m \times m}$ such that $Z^{2}=-I$,

$$
S(T)=\left[\begin{array}{ll}
0 & 0 \\
0 & Z
\end{array}\right], \quad Y=\left[\begin{array}{ll}
Y_{1} & 0
\end{array}\right],
$$

and $\frac{1}{2} Y_{1}$ is unitary.

Notice that the utility of the obtained results can be found in the manipulation of smaller exponents and matrices of smaller sizes than the original ones.

3. An algorithm to check the $\{k+1\}$-potency of $H(A)$. In this section we design an algorithm to decide whether $H(A)$ is $\{k+1\}$-potent when $k$ is odd, by using an SVD of a matrix $A$.

Let $A=\sum_{j=1}^{r} \sigma_{j} u_{j} v_{j}^{*}$ be a (reduced) SVD of a nonzero matrix $A \in \mathbb{C}^{n \times n}$ with $\left\{u_{1}, \ldots, u_{r}\right\}$ and $\left\{v_{1}, \ldots, v_{r}\right\}$ two orthonormal sets of column vectors of $\mathbb{C}^{n}$ and $\sigma_{1} \geq$ $\sigma_{2} \geq \cdots \geq \sigma_{r}>0$. It is well known that $\operatorname{rank}(A)=r$ and $\mathcal{N}(A)^{\perp}=\operatorname{span}\left\{v_{1}, \ldots, v_{r}\right\}$. Defining

$$
U_{r}=\left[\begin{array}{lll}
u_{1} & \cdots & u_{r}
\end{array}\right], \quad V_{r}=\left[\begin{array}{lll}
v_{1} & \cdots & v_{r}
\end{array}\right], \quad \Sigma=\operatorname{diag}\left(\sigma_{1}, \ldots, \sigma_{r}\right)
$$

we have that $A=U_{r} \Sigma V_{r}^{*}$, which implies that $A V_{r}=U_{r} \Sigma$ because

$$
U_{r}^{*} U_{r}=I, \quad V_{r}^{*} V_{r}=I .
$$


Notice that, in general, $U_{r}$ and $V_{r}$ are not unitary matrices.

ALGORITHM: This algorithm decides whether $H(A)$ is $\{k+1\}$-potent when $k$ is odd.

Input: A square matrix $A$.

Step 1. Compute a (reduced) SVD of $A$ and set $U_{r}, V_{r}$, and $\Sigma$ as before.

Step 2. Compute $M=V_{r}^{*} U_{r}$.

Step 3. If $U_{r}=V_{r} M$ and $H(M \Sigma)^{2}=H(M \Sigma)$ then $H(A)^{k+1}=H(A)$ for all $k \in \mathbb{N}$, else $H(A)^{k+1} \neq H(A)$ for all odd $k \in \mathbb{N}$.

Before we proceed to justify this algorithm, we illustrate it with an example by using MATLAB R2010b (Version 7.11.0.584). Let

$$
A=\frac{1}{15}\left[\begin{array}{rrrr}
1 & 2 & 3 & -1 \\
2 & 4 & 6 & -2 \\
3 & 6 & 9 & -3 \\
-1 & -2 & -3 & 1
\end{array}\right]
$$

An SVD of $A$ is

$$
U_{r}=\left[\begin{array}{r}
-0.2582 \\
-0.5164 \\
-0.7746 \\
0.2582
\end{array}\right], \quad \Sigma=1.0000, \quad V_{r}=\left[\begin{array}{r}
-0.2582 \\
-0.5164 \\
-0.7746 \\
0.2582
\end{array}\right] .
$$

Since $M=V_{r}^{*} U_{r}=1$ and $H(M \Sigma)^{2}=H(M \Sigma)$, then we get $H(A)^{k+1}=H(A)$ for all $k \in \mathbb{N}$.

We now consider a matrix $R=\left[\begin{array}{lll}v_{r+1} & \cdots & v_{n}\end{array}\right]$ whose columns form an orthonormal basis of $\mathcal{N}(A)$, that is $A R=0$. Define the unitary matrix $V=\left[\begin{array}{ll}V_{r} & R\end{array}\right]$. Now we get

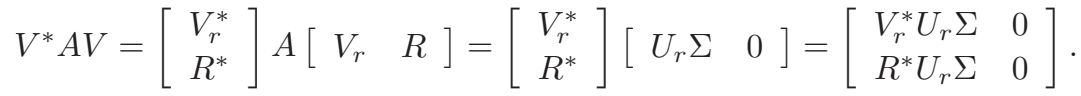

Set $T=V_{r}^{*} U_{r} \Sigma \in \mathbb{C}^{r \times r}$ and $Y=R^{*} U_{r} \Sigma \in \mathbb{C}^{(n-r) \times r}$. Hence

$$
A=V\left[\begin{array}{ll}
T & 0 \\
Y & 0
\end{array}\right] V^{*}
$$

that is, $A$ has the form (2.1). In the sequel we recall Remark 2.2. There exist a unitary matrix $W \in \mathbb{C}^{r \times r}$ and a diagonal (positive definite) matrix $E \in \mathbb{C}^{m \times m}$ such that $Y^{*} Y=W \operatorname{diag}(E, 0) W^{*}$. Let $T_{N}=W^{*} T W \in \mathbb{C}^{r \times r}, Y_{N}=Y W \in \mathbb{C}^{(n-r) \times r}$, 
and $V_{N}=V \operatorname{diag}(W, I) \in \mathbb{C}^{n \times n}$. Then

$$
A=V_{N}\left[\begin{array}{ll}
T_{N} & 0 \\
Y_{N} & 0
\end{array}\right] V_{N}^{*},
$$

where $Y_{N}^{*} Y_{N}=\operatorname{diag}(E, 0)$.

Suppose $H(A)^{k+1}=H(A)$ for some odd $k \in \mathbb{N}$. We apply part (I)-(a) of Theorem 2.6 and we conclude:

- $Y_{N}=0$, that is, $R^{*} U_{r}=0$. Hence, $v_{i}^{*} u_{j}=0$ for $r+1 \leq i \leq n$ and $1 \leq j \leq r$. In other words, $u_{1}, \ldots, u_{r}$ are orthonormal to $v_{r+1}, \ldots, v_{n}$, and thus $u_{1}, \ldots, u_{r} \in \operatorname{span}\left\{v_{1}, \ldots, v_{r}\right\}$. Therefore, there exists $M \in \mathbb{C}^{r \times r}$ such that

$$
U_{r}=V_{r} M
$$

Multiplying (3.2) by $V_{r}^{*}$ from the left and using (3.1), we deduce $M=V_{r}^{*} U_{r}$. Inserting this in (3.2), we get $U_{r}=V_{r} V_{r}^{*} U_{r}$.

- $H\left(T_{N}\right)$ is idempotent, which is equivalent to $H\left(V_{r}^{*} U_{r} \Sigma\right)$ is idempotent.

We now suppose that $U_{r}=V_{r} V_{r}^{*} U_{r}$ holds and $H\left(V_{r}^{*} U_{r} \Sigma\right)$ is idempotent. By Theorem 2.6, the algorithm will be justified if we check that $Y_{N}=0$. This fact follows from

$$
Y_{N}=R^{*} U_{r} \Sigma W=R^{*} V_{r} V_{r}^{*} U_{r} \Sigma W=0
$$

since $R^{*} V_{r}=0$.

We can also deduce the following result.

Proposition 3.1. Let $A \in \mathbb{C}^{n \times n}$ and $k \in \mathbb{N}$ be odd. If $H(A)$ is $\{k+1\}$-potent then $A$ is an EP matrix.

Proof. By the above, we have that $U_{r}=V_{r} V_{r}^{*} U_{r}$, that is, $\left(I-V_{r} V_{r}^{*}\right) U_{r}=0$, which implies $\mathcal{R}\left(U_{r}\right) \subseteq \mathcal{N}\left(I-V_{r} V_{r}^{*}\right) \subseteq \mathcal{R}\left(V_{r} V_{r}^{*}\right) \subseteq \mathcal{R}\left(V_{r}\right)$. The considered SVD assures that $\mathcal{R}\left(U_{r}\right)=\mathcal{R}(A)$ and $\mathcal{R}\left(V_{r}\right)=\mathcal{R}\left(A^{*}\right)$. Now we have $\mathcal{R}(A) \subseteq \mathcal{R}\left(A^{*}\right)$. Since $A$ and $A^{*}$ have the same rank, we also have $\mathcal{R}\left(A^{*}\right) \subseteq \mathcal{R}(A)$. Hence $A$ is an $E P$ matrix.

We end this section noticing that similar results can be obtained for $S(A)$.

4. Relation with the normality property. Groß showed in [14] that there are matrices $A$ such that $H(A)^{2}=H(A)$ and $A$ is not normal. The matrix $A$ in Example 2.7 is such that $H(A)^{3}=H(A)$ and $A$ is not normal.

In this section we shall present some relations between the $\{k+1\}$-potency of 
$H(A)$, respectively $S(A)$, and the normality of $A$.

Lemma 4.1. Let $T \in \mathbb{C}^{n \times n}$ be an upper triangular matrix. If $H(T)^{3}=H(T)$ and the real part of the eigenvalues of $T$ is in $\{-1,1\}$ then $T$ is diagonal.

Proof. It follows by induction on the size of the matrix $T$. If $n=1$, the conclusion is evident. Suppose $n>1$ and that the result is valid for every $(n-1) \times(n-1)$ matrix. Let

$$
T=\left[\begin{array}{cc}
\lambda_{1} & t_{12} \\
0 & T_{22}
\end{array}\right] \in \mathbb{C}^{n \times n},
$$

with $\lambda_{1} \in \mathbb{C}, t_{12} \in \mathbb{C}^{1 \times(n-1)}, T_{22} \in \mathbb{C}^{(n-1) \times(n-1)}$, be such that $H(T)^{3}=H(T)$ and $T_{22}$ is upper triangular. Then

$$
H(T)=\left[\begin{array}{cc}
\operatorname{Re} \lambda_{1} & \frac{1}{2} t_{12} \\
\frac{1}{2} t_{12}^{*} & H\left(T_{22}\right)
\end{array}\right],
$$

so we have that the entry $(1,1)$ in $H(T)^{2}$ is equal to $\left(\operatorname{Re} \lambda_{1}\right)^{2}+\frac{1}{4} t_{12} t_{12}^{*}$. The real part of the eigenvalues of $T$ is in $\{-1,1\}$, thus $\left(\operatorname{Re} \lambda_{1}\right)^{2}=1$. Hence, the entry $(1,1)$ in $H(T)^{2}$ is equal to $1+\frac{1}{4} t_{12} t_{12}^{*}$.

Since $H(T)^{3}=H(T)$, the eigenvalues of $H(T)$ are in the set $\{-1,0,1\}$. Then the eigenvalues of $H(T)^{2}$ are in the set $\{0,1\}$. Thus there exists a unitary $U \in \mathbb{C}^{n \times n}$ such that

$$
H(T)^{2}=U\left[\begin{array}{cc}
I_{r} & 0 \\
0 & 0
\end{array}\right] U^{*}
$$

with $r \leq n$. If we write

$$
U=\left[\begin{array}{ll}
U_{1} & U_{2} \\
U_{3} & U_{4}
\end{array}\right]
$$

with $U_{1} \in \mathbb{C}^{r \times r}, U_{2} \in \mathbb{C}^{r \times(n-r)}, U_{3} \in \mathbb{C}^{(n-r) \times r}, U_{4} \in \mathbb{C}^{(n-r) \times(n-r)}$, then

$$
H(T)^{2}=\left[\begin{array}{cc}
U_{1} U_{1}^{*} & U_{1} U_{3}^{*} \\
U_{3} U_{1}^{*} & U_{3} U_{3}^{*}
\end{array}\right]
$$

Since $U$ is unitary, we have $U_{1} U_{1}{ }^{*}+U_{2} U_{2}{ }^{*}=I$. If $U_{1}=\left[u_{i j}\right]$ and $U_{2}=\left[v_{i j}\right]$, then comparing the entries $(1,1)$ we get $\sum_{j=1}^{r}\left|u_{1 j}\right|^{2}+\sum_{j=1}^{n-r}\left|v_{1 j}\right|^{2}=1$. Since the entry $(1,1)$ in $H(T)^{2}$ is equal to $\sum_{j=1}^{r}\left|u_{1 j}\right|^{2}$, now we have that it is also equal to $1-\sum_{j=1}^{n-r}\left|v_{1 j}\right|^{2}$.

Finally, we have $1+\frac{1}{4} t_{12} t_{12}^{*}=1-\sum_{j=1}^{n-r}\left|v_{1 j}\right|^{2}$, that is, $\frac{1}{4} t_{12} t_{12}^{*}+\sum_{j=1}^{n-r}\left|v_{1 j}\right|^{2}=0$. This implies $t_{12}=0$. By induction hypothesis, $T_{22}$ is diagonal and then $T$ is diagonal as well.

Theorem 4.2. Let $A \in \mathbb{C}^{n \times n}$ and $k \in \mathbb{N}$. 
(I) Suppose $k$ is odd and consider the following statements:

(a) $H(A)^{k+1}=H(A)$.

(a') $H(A)^{k}=I$.

(b) The real part of the eigenvalues of $A$ is in $\{0,1\}$.

(b') The real part of the eigenvalues of $A$ is 1 .

(c) A is a normal matrix.

Then the following implications hold: $(\mathrm{a}) \wedge(\mathrm{b}) \Rightarrow(\mathrm{c}), \quad(\mathrm{a}) \wedge(\mathrm{c}) \Rightarrow(\mathrm{b}), \quad(\mathrm{b}) \wedge(\mathrm{c})$ $\Rightarrow(\mathrm{a}), \quad(\mathrm{a}) \wedge\left(\mathrm{b}^{\prime}\right) \Leftrightarrow\left(\mathrm{a}^{\prime}\right) \wedge(\mathrm{c}), \quad\left(\mathrm{b}^{\prime}\right) \wedge(\mathrm{c}) \Rightarrow\left(\mathrm{a}^{\prime}\right)$.

(II) Suppose $k$ is even and consider the following statements:

(a) $H(A)^{k+1}=H(A)$.

(a') $H(A)^{k}=I$.

(b) The real part of the eigenvalues of $A$ is in $\{-1,0,1\}$.

(b') The real part of the eigenvalues of $A$ is in $\{-1,1\}$.

(c) $A$ is a normal matrix.

Then the following implications hold: $(\mathrm{a}) \wedge(\mathrm{c}) \Rightarrow(\mathrm{b}), \quad(\mathrm{b}) \wedge(\mathrm{c}) \Rightarrow(\mathrm{a})$, $(\mathrm{a}) \wedge\left(\mathrm{b}^{\prime}\right) \Leftrightarrow\left(\mathrm{a}^{\prime}\right) \wedge(\mathrm{c}), \quad\left(\mathrm{b}^{\prime}\right) \wedge(\mathrm{c}) \Rightarrow\left(\mathrm{a}^{\prime}\right)$.

(III) If $k$ is not a multiple of 4 , then $S(A)^{k+1}=S(A)$ if and only if $A$ is Hermitian.

(IV) Suppose $k$ is a multiple of 4 and consider the following statements:

(a) $S(A)^{k+1}=S(A)$.

(a') $S(A)^{k}=I$.

(b) The imaginary part of the eigenvalues of $A$ is in $\{-1,0,1\}$.

(b') The imaginary part of the eigenvalues of $A$ is in $\{-1,1\}$.

(c) $A$ is a normal matrix.

Then the following implications hold: $(\mathrm{a}) \wedge(\mathrm{c}) \Rightarrow(\mathrm{b}), \quad(\mathrm{b}) \wedge(\mathrm{c}) \Rightarrow(\mathrm{a})$, $(\mathrm{a}) \wedge\left(\mathrm{b}^{\prime}\right) \Leftrightarrow\left(\mathrm{a}^{\prime}\right) \wedge(\mathrm{c}), \quad\left(\mathrm{b}^{\prime}\right) \wedge(\mathrm{c}) \Rightarrow\left(\mathrm{a}^{\prime}\right)$.

Proof. If $A$ is normal then it is unitarily similar to a diagonal matrix with the eigenvalues of $A$ as diagonal entries. Thus, without loss of generality, we can assume that $A=\operatorname{diag}\left(\lambda_{1}, \ldots, \lambda_{n}\right)$. Then $H(A)=\operatorname{diag}\left(\operatorname{Re} \lambda_{1}, \ldots, \operatorname{Re} \lambda_{n}\right)$ and $S(A)=$ $\operatorname{diag}\left(\operatorname{Im} \lambda_{1}, \ldots, \operatorname{Im} \lambda_{n}\right)$. It is now easy to see that $(\mathrm{a}) \wedge(\mathrm{c}) \Rightarrow(\mathrm{b}),(\mathrm{b}) \wedge(\mathrm{c}) \Rightarrow$ (a), $\left(a^{\prime}\right) \wedge(c) \Rightarrow(a) \wedge\left(b^{\prime}\right)$, and $\left(b^{\prime}\right) \wedge(c) \Rightarrow\left(a^{\prime}\right)$ in $(I),(I I)$, and $(I V)$.

Assume (a) and (b) in (I). Lemma 1.1 implies $H(A)^{2}=H(A)$, thus it remains to apply [14, Theorem 2] to conclude that $A$ is a normal matrix. Hence, $(\mathrm{a}) \wedge(\mathrm{b}) \Rightarrow(\mathrm{c})$ in $(\mathrm{I})$.

If (a) and (b') in (I) hold then (a) and (b) also hold. We have proved that then (c) holds as well. Furthermore, since $\left(b^{\prime}\right) \wedge(c) \Rightarrow\left(a^{\prime}\right)$ in (I), we have that (a') also holds.

Now assume (a) and (b') in (II). According to Lemma 1.1, we have $H(A)^{3}=$ $H(A)$. In view of Schur's triangularization theorem, $A$ is unitarily similar to an upper triangular matrix $T$. Since $H(T)^{3}=H(T)$, Lemma 4.1 implies that $T$ is diagonal, 
thus $A$ is normal. In particular, since (b') and (c) hold, we conclude that (a') holds as well. In other words, $(\mathrm{a}) \wedge\left(\mathrm{b}^{\prime}\right) \Rightarrow\left(\mathrm{a}^{\prime}\right) \wedge(\mathrm{c})$ in $(\mathrm{II})$.

Assume (a) and (b') in (IV). Then the real part of the eigenvalues of i $A$ is in $\{-1,1\}$ and $H(\mathrm{i} A)^{k+1}=H(\mathrm{i} A)$, so it remains to apply $(\mathrm{a}) \wedge\left(\mathrm{b}^{\prime}\right) \Rightarrow\left(\mathrm{a}^{\prime}\right) \wedge(\mathrm{c})$ in (II) to conclude $\left(\mathrm{a}^{\prime}\right) \wedge(\mathrm{c})$ in $(\mathrm{IV})$.

Finally, if $k$ is not a multiple of 4 then $S(A)^{k+1}=S(A)$ if and only if $S(A)=0$, by Lemma 1.1. Hence, (III) holds.

REMARK 4.3. The implications $(\mathrm{a}) \wedge(\mathrm{b}) \Rightarrow(\mathrm{c})$ in (II) and (IV) are not true in general. It is sufficient to observe the matrix

$$
A=\left[\begin{array}{ll}
0 & 0 \\
2 & 0
\end{array}\right] \text {. }
$$

Then we have $H(A)^{3}=H(A)$ and the real part of the eigenvalues of $A$ is 0 , that is, (a) and (b) in (II) hold. We also have $S(A)^{5}=S(A)$ and the imaginary part of the eigenvalues of $A$ is 0 , that is, (a) and (b) in (IV) hold. However, $A$ is not normal.

It is not difficult to verify that Theorem 4.2 implies the following result.

Corollary 4.4. Let $A \in \mathbb{C}^{n \times n}$ and let $k_{1}, k_{2} \in \mathbb{N}$. Consider the following statements:

(a) $A$ is normal.

( $\left.\mathrm{b}_{1}\right) H(A)^{k_{1}+1}=H(A)$ and $S(A)^{k_{2}+1}=S(A)$.

$\left(\mathrm{b}_{2}\right) H(A)^{k_{1}}=I$ and $S(A)^{k_{2}}=I$.

$\left(\mathrm{c}_{1}\right) \sigma(A) \subseteq\{0,1\}$.

$\left(\mathrm{c}_{2}\right) \sigma(A) \subseteq\{0,1, \mathrm{i}, 1+\mathrm{i},-\mathrm{i}, 1-\mathrm{i}\}$.

$\left(\mathrm{c}_{3}\right) \sigma(A) \subseteq\{-1,0,1\}$.

( $\left.\mathrm{c}_{4}\right) \sigma(A) \subseteq\{-1+\mathrm{i}, \mathrm{i}, 1+\mathrm{i},-1,0,1,-1-\mathrm{i},-\mathrm{i}, 1-\mathrm{i}\}$.

$\left(\mathrm{c}_{5}\right) \sigma(A) \subseteq\{1+\mathrm{i}, 1-\mathrm{i}\}$.

( $\left.\mathrm{c}_{6}\right) \sigma(A) \subseteq\{-1+\mathrm{i}, 1+\mathrm{i},-1-\mathrm{i}, 1-\mathrm{i}\}$.

$\left(\mathrm{d}_{1}\right) k_{1}$ is odd and $k_{2}$ is not a multiple of 4 .

$\left(\mathrm{d}_{2}\right) k_{1}$ is odd and $k_{2}$ is a multiple of 4 .

$\left(\mathrm{d}_{3}\right) k_{1}$ is even and $k_{2}$ is not a multiple of 4 .

$\left(\mathrm{d}_{4}\right) k_{1}$ is even and $k_{2}$ is a multiple of 4 .

Then the following implications hold:

$(\mathrm{a}) \wedge\left(\mathrm{b}_{1}\right) \wedge\left(\mathrm{d}_{i}\right) \Rightarrow\left(\mathrm{c}_{i}\right)$ for $i=1,2,3,4$;

$(\mathrm{a}) \wedge\left(\mathrm{c}_{i}\right) \wedge\left(\mathrm{d}_{i}\right) \Rightarrow\left(\mathrm{b}_{1}\right)$ for $i=1,2,3,4$;

$(\mathrm{a}) \wedge\left(\mathrm{b}_{2}\right) \wedge\left(\mathrm{d}_{2}\right) \Rightarrow\left(\mathrm{c}_{5}\right)$;

$(\mathrm{a}) \wedge\left(\mathrm{b}_{2}\right) \wedge\left(\mathrm{d}_{4}\right) \Rightarrow\left(\mathrm{c}_{6}\right)$; 


$$
\begin{aligned}
& (\mathrm{a}) \wedge\left(\mathrm{c}_{5}\right)+\left(\mathrm{d}_{2}\right) \Rightarrow\left(\mathrm{b}_{2}\right) ;(\mathrm{a}) \wedge\left(\mathrm{c}_{6}\right) \wedge\left(\mathrm{d}_{4}\right) \Rightarrow\left(\mathrm{b}_{2}\right) ; \\
& \left(\mathrm{b}_{1}\right) \wedge\left(\mathrm{c}_{5}\right) \wedge\left(\mathrm{d}_{2}\right) \Rightarrow(\mathrm{a}) \wedge\left(\mathrm{b}_{2}\right) ;\left(\mathrm{b}_{1}\right) \wedge\left(\mathrm{c}_{6}\right) \wedge\left(\mathrm{d}_{4}\right) \Rightarrow(\mathrm{a}) \wedge\left(\mathrm{b}_{2}\right)
\end{aligned}
$$

5. Final remarks. As we have pointed out in the introduction, the Hermitian part of a matrix is used in applications. For example, for a non-Hermitian positive definite linear system, the Hermitian and skew-Hermitian parts of its coefficient matrix were used to design generalized conjugate gradient method by Concus and Golub in [10], and they were also used to construct inner-outer iteration methods by Axelsson, Bai, and Qiu in [1].

For a Hermitian matrix $X$, we denote by $X>0$ the fact that $X$ is positive definite and by $X \geq 0$ that $X$ is positive semidefinite. We end this paper with a characterization for the positive semidefiniteness of $H(A)$.

Let $X \in \mathbb{C}^{n \times n}$ be a Hermitian matrix and suppose that there exist $r<n$, a Hermitian matrix $A \in \mathbb{C}^{r \times r}$, and a matrix $B \in \mathbb{C}^{(n-r) \times r}$ of rank $m$ such that $B^{*} B$ is diagonal with positive entries in the first $m$ diagonal positions and

$$
X=\left[\begin{array}{cc}
A & B^{*} \\
B & 0
\end{array}\right] \text {. }
$$

We shall prove that $X \geq 0$ if and only if $A \geq 0$ and $B=0$; this implies that $X>0$ cannot occur.

Namely, if $X \geq 0$ then there exists a matrix $Y \in \mathbb{C}^{n \times n}$ such that $X=Y^{*} Y$. Partitioning

$$
Y=\left[\begin{array}{ll}
C & D \\
E & F
\end{array}\right]
$$

in blocks of adequate sizes, we get

$$
\left[\begin{array}{cc}
A & B^{*} \\
B & 0
\end{array}\right]=\left[\begin{array}{cc}
C^{*} C+E^{*} E & C^{*} D+E^{*} F \\
D^{*} C+F^{*} E & D^{*} D+F^{*} F
\end{array}\right] .
$$

We conclude that $D=0$ and $F=0$, which implies $B=0$ and $A=C^{*} C+E^{*} E \geq 0$.

It is easy to see that $X \geq 0$ if and only if $U^{*} X U \geq 0$ for all unitary matrices $U$. By the above, the following proposition holds. Similar results were obtained in [2].

Proposition 5.1. Let $A \in \mathbb{C}^{n \times n}$ be a matrix of rank $r<n$. Then $H(A) \geq 0$ if and only if there exists a unitary matrix $U \in \mathbb{C}^{n \times n}$ such that $A$ has the form (2.1) with $H(T) \geq 0$ and $Y=0$. In particular, $H(A) \geq 0$ implies that $A$ is an EP matrix.

Acknowledgements. The authors would like to thank the referees for their valuable comments and suggestions. 


\section{REFERENCES}

[1] O. Axelsson, Z.-Z. Bai, S.-X. Qiu. A class of nested iteration schemes for linear systems with a coefficient matrix with a dominant positive definite symmetric part, Numer. Algorithms 35 (2004), 351-372.

[2] Z.-Z. Bai. On semi-convergence of Hermitian and skew-Hermitian splitting methods for singular linear systems, Computing 89 (2010), 171-197.

[3] Z.-Z. Bai, G.H. Golub, C.-K. Li. Optimal parameter in Hermitian and skew-Hermitian splitting method for certain two-by-two block matrices, SIAM J. Sci. Comput. 28 (2006), 583-603.

[4] Z.-Z. Bai, G.H. Golub, C.-K. Li. Convergence properties of preconditioned Hermitian and skew-Hermitian splitting methods for non-Hermitian positive semidefinite matrices, Math. Comp. 76 (2007), 287-298.

[5] Z.-Z. Bai, G.H. Golub, L.-Z. Lu, J.-F. Yin. Block triangular and skew-Hermitian splitting methods for positive-definite linear systems, SIAM J. Sci. Comput. 26 (2005), 844-863.

[6] Z.-Z. Bai, G.H. Golub, M.K. Ng. Hermitian and skew-Hermitian splitting methods for nonHermitian positive definite linear systems, SIAM J. Matrix Anal. Appl. 24 (2003), 603626.

7] Z.-Z. Bai, G.H. Golub, M.K. Ng. On successive-overrelaxation acceleration of the Hermitian and skew-Hermitian splitting iterations, Numer. Linear Algebra Appl. 14 (2007), 319-335.

8] Z.-Z. Bai, G.H. Golub, J.-Y. Pan. Preconditioned Hermitian and skew-Hermitian splitting methods for non-Hermitian positive semidefinite linear systems, Numer. Math. 98 (2004), $1-32$.

[9] O.M. Baksalary, G.P.H. Styan, G. Trenkler. On a matrix decomposition of Hartwig and Spindelböck, Linear Algebra Appl. 430 (2009), 2798-2812.

[10] P. Concus, G.H. Golub. A generalized conjugate gradient method for nonsymmetric systems of linear equations, in Computing Methods in Applied Sciences and Engineering, Lecture Notes in Econom. and Math. Systems, 134, R. Glowinski and J.R. Lions, eds., SpringerVerlag, Berlin, 1976, pp. 56-65.

[11] M. Benzi, M.J. Gander, G.H. Golub. Optimization of the Hermitian and skew-Hermitian splitting iteration for saddle-point problems, BIT 43 (2003), suppl., 881-900.

[12] M. Benzi, G.H. Golub, A preconditioner for generalized saddle point problems, SIAM J. Matrix Anal. Appl. 26 (2004), 20-41.

[13] M. Chacron, I.N. Herstein. Powers of skew and symmetric elements in division rings, Houston J. Math. 1 (1975), 15-27.

[14] J. Groß. Idempotency of the Hermitian part of a complex matrix, Linear Algebra Appl. 289 (1999), 135-139

[15] J. Groß. On singular periodic matrices, In: B. Schipp, W. Krämer (eds.), Statistical Inference, Econometric Analysis and Matrix Algebra, Festschrift in Honour of Götz Trenkler, PhysicaVerlag, Heidelberg, 2009, pp. 325-332.

[16] R.E. Hartwig, K. Spindelböck. Matrices for which $A^{*}$ and $A^{\dagger}$ commute, Linear and Multilinear Algebra 14 (1984), 241-256.

[17] M. Ho. Iterative methods for non-hermitian positive semi-definite systems, Ph.D. Thesis, The University of Hong Kong Press, 2004.

[18] R.A. Horn, C.R. Johnson. Matrix Analysis, Cambridge University Press, Cambridge, 1985.

[19] C.G. Khatri. Powers of matrices and idempotency, Linear Algebra Appl. 33 (1980), 57-65.

[20] M.-Q. Jiang, Y. Cao, On local Hermitian and skew-Hermitian splitting iteration methods for generalized saddle point problems, J. Comput. Appl. Math. 231 (2009), 973-982.

[21] J. Li, Q. Zhang, S. Wu. Semi-convergence of the local Hermitian and skew-Hermitian splitting iteration methods for singular generalized saddle point problems, Appl. Math. E-Notes 11 (2011), 82-90.

[22] R. Mathias. Matrices with positive definite Hermitian part: inequalities and linear systems, 
Electronic Journal of Linear Algebra ISSN 1081-3810

A publication of the International Linear Algebra Society

Volume 24, pp. 95-112, June 2012

SIAM J. Matrix Anal. Appl. 13 (1992), 640-654.

[23] J.P. McCloskey. Characterizations of $r$-potent matrices, Math. Proc. Cambridge Philos. Soc. 96 (1984), 213-222.

[24] J.P. McCloskey. Properties of normal, $r$-potent matrices, Linear and Multilinear Algebra 17 (1985), 325-336. 